\title{
The Efficacy of Electroconvulsive Therapy in Major Depressive Disorder Relapsed or Unresponsive to Transcranial Magnetic Stimulation in Pregnancy: Three Case Studies
}

\author{
Eylem Ozten $^{1}$, Gokben Hızlı Sayar ${ }^{1, *}$, Işıl Gögcegöz Gül ${ }^{1}$, Ali Evren Tufan ${ }^{2}$, Cem Cerit ${ }^{3}$, Nesrin Dilbaz ${ }^{1}$ \\ ${ }^{1}$ Uskudar University Medical Faculty, Department of Psychiatry, Istanbul, Turkey \\ ${ }^{2}$ AbantIzzetBaysal University Medical Faculty, Department of Child and Adolescent Psychiatry, Bolu, Turkey \\ ${ }^{3}$ Kocaeli University Medical Faculty, Department of Psychiatry, Kocaeli, Turkey \\ *Corresponding author: gokben.hizlisayar@uskudar.edu.tr
}

Received January 08, 2014; Revised January 13, 2014; Accepted January 16, 2014

\begin{abstract}
About 5-10\% of women experience depression during pregnancy. The potentially adverse effects of pharmacological treatment (e.g. teratogenicity, toxicity, foetal developmental abnormalities and withdrawal symptoms) on both the mother and the foetus mean that psychopharmacological treatments have limited application for pregnant women. In these cases treatment needs to be tailored to the individual patient and non-pharmacological options may be appropriate. Transcranial Magnetic Stimulation (TMS) and Electroconvulsive treatment (ECT) may be viable alternatives for these patients. We report on three patients in their first trimester of pregnancy suffering from major depressive disorder (MDD). All three were initially treated with TMS and psychotherapy because of the reduced risk of side effects. Two patients failed to respond adequately to treatment and were switched to ECT. The third patient displayed an adequate response to TMS but failed to maintain the improvement. This patient had to receive ECT in the post-partum period due to on-going depressive symptoms. Subsequently this patient responded adequately to ECT. Further studies of novel, non-pharmacological treatment methods for MDD during pregnancy (such as TMS and ECT) and detailed investigations of the factors associated with treatment responses in such patients are needed. Our cases demonstrate the need to evaluate the effectiveness of TMS in larger samples of pregnant patients diagnosed with MDD.
\end{abstract}

Keywords: electroconvulsive treatment, major depressive disorder, pregnancy, transcranial magnetic stimulation

Cite This Article: Eylem Ozten, Gokben Hızıı Sayar, Işıl Gögcegöz Gül, Ali Evren Tufan, Cem Cerit, and Nesrin Dilbaz, "The Efficacy of Electroconvulsive Therapy in Major Depressive Disorder Relapsed or Unresponsive to Transcranial Magnetic Stimulation in Pregnancy: Three Case Studies.” American Journal of Clinical Medicine Research 2, no. 1 (2014): 18-21. doi: 10.12691/ajcmr-2-1-5.

\section{Introduction}

Untreated prenatal depression has negative impacts on both mother and baby. These impacts include inadequate weight gain, preeclampsia, preterm delivery, reduced birth weight, reduced head size, and negative outcomes for infant neuropsychological development and social behavior during adolescence [1,2]. Moreover, depression during pregnancy may continue during the post-partum period. The risks of untreated maternal depression include emotional dysregulation, social and cognitive problems and insecure maternal-infant attachment that may lead to behavioral and relational problems in later life [3,4,5].

The prevalence of major depressive disorder (MDD) among pregnant women and the impact of its consequences on both the mother and her infant underline the importance of identifying treatments which are effective and safe. Recent guidelines published by the
American Psychiatric Association (APA) recommend psychotherapy as the treatment of choice for mild and moderate depression in pregnant women and suggest that antidepressants be used for those with moderate to severe depression. Treating only perinatal depression may not be sufficient in protecting children against poor long-term outcomes. Thus psychotherapy of perinatal depression should also include an approach focused on the parenting role. Its effectiveness concerns not only the improvement of the mother's symptoms but also the quality of the parent-infant relationship. However, the limited number and availability of clinicians experienced in providing psychotherapy to this specific population as well as the partial response of symptoms to therapy can pose problems [1].

When a patient's fails to enter into remission following initial treatment with antidepressants several other choices are available. These include pharmacological options such as switching to a different class of antidepressant, 
combining the existing antidepressant with a second antidepressant or augmenting the antidepressant with a non-antidepressant agent. Alternatively, a non-invasive treatment such as repetitive Transcranial Magnetic Stimulation (rTMS) can be applied [6]. rTMS involves neuromodulation via delivery of focused magnetic pulses to targeted areas of the cerebral cortex. The United States' Food and Drug Administration cleared the use of the rTMS system for the treatment of MDD in adult patients with depression who, "failed to achieve satisfactory improvement from one prior antidepressant medication at or above the minimal effective dose and duration in the current episode" [7].

Case studies of the reliability and efficacy of rTMS in pregnant women suffering from depression are currently scarce. Zhang and colleagues reported that rTMS was helpful for three pregnant women with depression and had no adverse effects on the foetus [8]. Nahas et al. reported a case of depression in pregnancy which was treated successfully with rTMS [9]. The largest study of the application of rTMS to pregnant women with depression was carried out by Kim et al. whose sample consisted of 10 such patients. They reported that $70 \%$ of the sample responded positively to treatment and there were no side effects either on the foetus or the mother [10].

The expert consensus guidelines published by the APA suggest that ECT is safe in all trimesters of pregnancy [11] An extensive literature review was carried out by Anderson and Reti. Their study spanned the period 19412007 and consisted of 339 patients who received ECT while pregnant. They found that $84 \%$ of patients achieved at least partial remission [12]. In addition their results suggested that the prevalence of adverse foetal effects that could be directly related to maternal ECT was 3.2\% [13].

We report on three patients in their first trimester of pregnancy that fulfilled DSM-IV-TR criteria for MDD. These patients were initially treated with rTMS but subsequently had to treated with ECT either due to an inadequate response $(n=2)$ or the failure to maintain treatment effects $(n=1)$. The socio-demographic and clinical features of the three patients are given in Table 1.

Table 1. Socio-demographic and clinical features of patients

\begin{tabular}{|l|l|l|l|}
\hline & Patient I & Patient II & Patient III \\
\hline Age & 25 & 30 & 32 \\
\hline Presenting symptoms & $\begin{array}{l}\text { apathy, insomnia, sadness, crying, } \\
\text { ruminations about health of baby }\end{array}$ & $\begin{array}{l}\text { apathy, thoughts of death, insomnia, distress, } \\
\text { feelings of inadequacy }\end{array}$ & $\begin{array}{l}\text { apathy, distress, sadness, pain, } \\
\text { hopelessness }\end{array}$ \\
\hline Duration of symptoms & 7 weeks & 5 weeks & 6 weeks \\
\hline Previous treatments & fluoxetine & venlafaxine & $\begin{array}{l}\text { citalopram, } \\
\text { mirtazapine, TMS }\end{array}$ \\
\hline $\begin{array}{l}\text { Pregnancy } \\
\text { presentation }\end{array}$ & Second & First (twins) & Second \\
\hline HDRS-17 (Baseline) & 28 & 34 & 27 \\
\hline HDRS-17 (After TMS) & 26 & 38 & $6 / 34$ \\
\hline HDRS-17 (After ECT) & 8 & 6 & 8 \\
\hline Side effects of TMS & Headache & - & - \\
\hline $\begin{array}{l}\text { First Min. APGAR } \\
\text { Score }\end{array}$ & 10 & $8 / 10$ & 8 \\
\hline $\begin{array}{l}\text { Fifth Min. APGAR } \\
\text { Score }\end{array}$ & 10 & $10 / 10$ & 10 \\
\hline
\end{tabular}

HDRS-17: Hamilton Depression Rating Scale 17 Item, TMS: transcranial magnetic stimulation, ECT: electroconvulsive treatment

\section{Case Studies}

\subsection{Case 1}

A 25 year old woman had a seven year history of moderate to severe MDD. Her depressive symptoms had responded well to pharmacological treatment. Because of her desire to have another baby, she had stopped taking medication six months previously and her depressive symptoms had returned.

She had been taking fluoxetine $40 \mathrm{mg}$ /day for six years following her first episode. When she first presented she was clinically determined to be severely depressed, with depressed mood, loss of usual interests, hopelessness, feelings of guilt and frequent suicidal thoughts. The patient had been married for eight years and had been pregnant without gestational problems for seven weeks at the time of application. She had no history of mania, substance use disorder or medical illness. Following psychiatric evaluation, the patient was diagnosed with MDD. After providing information about the benefits and drawbacks of all the available treatment choices, informed consent to rTMS was given by the patient. Following consultation with her obstetrician she underwent a course of 20 rTMS treatments, six days per week together with supportive therapy over the left prefrontal cortex. She tolerated the treatment well except for local discomfort during the first week. After 20 treatments she continued to feel depression and fatigue and had crying spells. Her symptoms had not improved and she still had negative thoughts. Hamilton Depression Rating Scale (HDRS) scores are given in Table 1. As a result of the inadequate response she was hospitalized during the thirteenth week of pregnancy and ECT was planned. After informed consent was obtained, 10 sessions of ECT were applied. There were no adverse effects and the patient responded to treatment. She was subsequently discharged and followedup on an outpatient basis without relapse. She had a normal, spontaneous vaginal delivery in thirty-eighth week of her pregnancy. The baby weighed 3,600 grams at 
birth. The post-partum period was normal with no adverse consequences for the baby or the mother.

\subsection{Case 2}

The patient had been married for three years and was eight weeks pregnant with twins. Her complaint had begun five years earlier triggered by an interpersonal stressor. She had responded to venlafaxine $300 \mathrm{mg} / \mathrm{day}$ but had relapsed a month before she was seen by us. Her treatment had stopped six months earlier. She had no history of mania, substance use disorder or medical illness, but her mother and sister had a history of depression. The patient was diagnosed with MDD and had to be hospitalized because of suicidal thoughts. She chose rTMS after being informed of treatment options. After obtaining written informed consent and a consultation with an obstetrician, she received 20 sessions of rTMS along with psychotherapy. No significant side effects were observed as a result of the rTMS. HDRS scores are given in Table 1. Due to the lack of adequate response to treatment and following consultation with her obstetrician who again informed her of treatment options and obtained written informed consent she switched to ECT. She received and responded to 10 sessions. No gestational problems were observed and the patient's only complaint was slight and temporary forgetfulness. She was discharged in remission and no relapse was observed throughout pregnancy. She gave birth by caesarean section in the thirty-sixth week of her pregnancy to twins weighing 2,600 and 3,000 grams. No adverse consequences either for the mother or her children could be observed.

\subsection{Case 3}

The patient had been married for nine years and was 10 weeks pregnant without gestational problems. Symptoms had begun six years earlier during her first pregnancy. She began citalopram $20 \mathrm{mg} /$ day in the fifth month of pregnancy but did not benefit from treatment. Six months after her first pregnancy she conceived again. However, the pregnancy had to be terminated in the eighth week with her and her physician's agreement as a result of worsening depressive symptoms. She was treated with paroxetine $40 \mathrm{mg} /$ day and mirtazapine $30 \mathrm{mg} /$ day for two years but experienced a relapse four months after she stopped taking medication. During her second episode she received 15 sessions of rTMS together with paroxetine 20 $\mathrm{mg} /$ day. Her symptoms remained under control for the following two years, only reappearing following conception and cessation of paroxetine.

At the time of application she had been symptomatic for the previous six weeks. She had no history of mania, substance use disorder or medical illness; however her sister had a history of MDD. The patient was diagnosed with MDD and treatment with rTMS was planned, both because she was in her first trimester of pregnancy and because of she had previously responded to this form of treatment. After obtaining written informed consent and following consultation with an obstetrician, she received 20 sessions of rTMS together with psychotherapy. No significant side-effects were observed with rTMS. She was discharged and followed up on an outpatient basis. However, her symptoms reappeared in the thirty-second week of her pregnancy. Upon her request and because of her depressive symptoms, a healthy baby weighing 2,400 grams was delivered in the thirty-fourth week by caesarean section. She was hospitalized for a second time due to suicidal thoughts and her inability to feed the baby. Mirtazapine $30 \mathrm{mg} /$ day and paroxetine $40 \mathrm{mg} /$ day were restarted. Due to her suicidal ideation and after obtaining written informed consent she received 10 sessions of ECT. She responded to treatment and had maintained this remission at a 28 week follow-up.

The socio-demographic and clinical features of pregnant patients with MDD who were initially treated with TMS and switched to ECT are given in Table 1.

rTMS was applied using the Magstim Super Rapid stimulator (Magstim Company, Whitland, United Kingdom) with figure-of-eight shaped coil in all three patients. The rTMS intensity was set at $100 \%$ of motor threshold, determined by visual inspection. Stimulation was given to the left prefrontal cortex. The treatment schedule was six days per week. A $25 \mathrm{~Hz}$ stimulation lasting two seconds was delivered 20 times at 30-second intervals. A full course consisted of 1,000 magnetic pulses.

ECT was administered three times per week with a MECTA Spectrum 5000 Q (MECTA Corporation, Tualatin, Oregon). Bitemporal bilateral electrode placement was selected. The treatment team consisted of a psychiatrist, a psychiatric nurse, an anaesthesiologist, obstetrics and gynaecology nurse, and a specialist in internal medicine. Patients were intubated to ensure adequate ventilation. Foetal heart rate was monitored during the procedure. Bicitrate $30 \mathrm{~mL}$ was administered orally before induction to raise the gastric $\mathrm{pH}$ and reduce the risk of aspiration pneumonitis. Anaesthesia induction was attained with methohexital 100-150 mg IV and succinylcholine 100-120 mg IV. Seizure threshold was determined using a dose titration schedule.

\section{Discussion}

Untreated depression during pregnancy has been associated with increased rates of adverse maternal, obstetrical and fetal outcomes; consequently, it is essential to manage these women effectively and adequately during this vulnerable time of their lives [14]. Pharmacological treatment during pregnancy requires balancing risks and expected benefits in each patient, taking the patients' history, presentation, and preferences into account. The safety of antidepressant use during pregnancy is still under discussion since studies on risks of serious malformations, persistent pulmonary hypertension, and long-term effects on neurodevelopment report conflicting results [15].

Food and Drug Administration have not approved any antidepressant medication to be used for pregnant women. Thus, it is crucial to consider alternative therapies for depressed women during pregnancy. rTMS is an effective and viable alternative treatment for pregnant women with depression. In a recent study safety and effectiveness of high-frequency rTMS assessed in antenatal depression. Thirty depressed pregnant patients received rTMS over the left prefrontal cortex. After the treatment period, $41.4 \%$ of the study group demonstrated significant mood improvements as indexed by a reduction of more than $50 \%$ on the HDRS score. In addition, $20.7 \%$ attained 
remission, 34.5\% achieved a partial response, and 3.4\% had worsening in HDRS scores at the end of treatment Treatment was well tolerated, and no significant adverse effects were reported [16].

Although rTMS was well tolerated and found to be statistically and clinically effective in pregnant patients with treatment resistant depression in those studies, our most significant finding was the low response to rTMS and the poor maintenance of treatment effects in the sole patient who did respond to rTMS. Although Kim and colleagues reported a $70 \%$ response rate in their patients, they also noted that in $60 \%$ of cases patients were using antidepressants at the time when TMS was applied [10]. Therefore, it can be argued that the limited efficacy and maintenance of treatment effects in our patients may be due to a lack of concurrent use of antidepressants. Previous studies typically averaged 15,000-30,000 total magnetic pulses over a course of TMS [17]. However, recent studies provide 60,000-90,000 pulses during rTMS course for treatment of depression [18]. In our report, subjects received 1000 stimulations/session for 20 sessions (20,000 pulses) at $100 \%$ of motor threshold. It may be considered as an inadequate treatment course. However, questions remain about the parameter optimization.

All three of our patients had to switch to ECT due to the lack of adequate response to TMS treatment or lack of maintenance. At the same time, none of our patients had any maternal or foetal side effects. This is consistent with both previous studies and the expert consensus guidelines published by the APA which suggests that ECT is safe in all trimesters of pregnancy [11].

Miller et al described 300 cases, found 28 instances of likely ECT-related complications, including transient benign fetal arrhythmias, mild vaginal bleeding, abdominal pain, and self-limited uterine contractions. Several more serious concerns including stillbirth, neonatal death, and congenital malformations were described, however, all were thought due to factors unrelated to the ECT [19]. Anderson and Reti reported 339 cases with 18 cases of ECT related complications in the mothers (including status epilepticus, preterm labor, vaginal bleeding, and placental abruption) and 20 cases in the fetuses, most typically arrhythmias. There was also a single case of fetal death, which was thought due to status epilepticus in the mother [12].

TMS is a viable option for the pregnant or postpartum patient with depression. Although its clinical efficacy does not compare well to that of ECT, it is significantly less invasive than either and avoids the systemic effects of pharmaceutical therapies. However, if the depression is severe, resistant, and requires immediate treatment, ECT is an excellent option in the peripartum patient.

\section{Conclusion}

The pregnancy period is important due to the fact that psychiatric disorders are common and treatment choices are limited. Therefore, studies of novel, nonpharmacological treatment methods for MDD during pregnancy (such as TMS and ECT) and investigations of the factors associated with treatment response in such patients are needed. Our case studies demonstrate the need to evaluate the effects of TMS in larger samples of pregnant patients diagnosed with MDD.

\section{References}

[1] Kurki T, Hiilesmaa V, Raitasalo R, et al. Depression and anxiety in early pregnancy and risk for preeclampsia. Obstet Gynecol. 2000; 95: 487-490.

[2] Nonacs R, Cohen LS. Assessment and treatment of depression during pregnancy: an update. PsychiatrClin North Am. 2003; 26: 547-562.

[3] Galler JR, Harrison RH, Ramsey F, et al. Maternal depressive symptoms affect infant cognitive development in Barbados. $J$ Child Psychol Psychiatry. 2000; 41 (6): 747-757.

[4] Newport DJ, Stowe ZN, Nemeroff CB. Parental depression: animal models of an adverse life event. Am J Psychiatry. 2002; 159 (8): 1265-1283.

[5] Deave T, Heron J, Evans J, et al. The impact of maternal depression in pregnancy on early child development. Br J Obstet Gynaecol. 2008; 115: 1043-1051.

[6] American Psychiatric Association. Practice Guideline for the Treatment of Patients With Major Depressive Disorder, Third Edition. Washington, DC: American Psychiatric Association; 2010. Available from

http://www.psychiatryonline.com/pracGuide/pracGuideTopic 7.aspx.

[7] Schutter DJ. Antidepressant efficacy of high-frequency transcranial magnetic stimulation over the left dorsolateral prefrontal cortex in double-blind sham-controlled designs: A meta-analysis. Psychol Med. 2009; 39: 65-75.

[8] Zhang D, Hu Z. RTMS may be a good choice for pregnant women with depression. Arch Women Ment Health. 2009; 12 (3): 189-190.

[9] Nahas Z, Bohning D, Molloy MA, et al. Safety and feasibility of repetitive transcranial magnetic stimulation in the treatment of anxious depression in pregnancy: a case report. J Clin Psychiatry. 1999; 60: 50-52.

[10] Kim DR, Epperson N, Pare E, et al. An open label pilot study of transcranial magnetic stimulation for pregnant women with major depressive disorder. J Women's Health. 2011; 20: 255-261.

[11] American Psychiatric Association. The Practice of Electroconvulsive Therapy: Recommendations for treatment, training and privileging: A task force report of the American Psychiatric Association. Second Edition, Washington DC: American Psychiatric Press; 2001.

[12] Anderson EL, Reti IM. ECT in pregnancy: A review of the literature from 1941 to 2007. Psychosomatic Medicine. 2009; 71 (2): 235-242.

[13] Tan O, Tarhan N, Coban A, et al. Antidepressant effect of 58 sessions of rTMS in a pregnant woman with recurrent major depressive disorder: A case report. Prim Care Companion J Clin Psychiatry. 2008; 10 (1): 69-71.

[14] Robakis TK, Williams, KE. Biologically based treatment approaches to the patient with resistant perinatal depression. Arch Womens Ment Health. 2013; 16: 343-351.

[15] Andrade SE, McPhillips H, Loren D, et al. Antidepressant medication use and risk of persistent pulmonary hypertension of the newborn. Pharmacoepidemiol Drug Saf. 2009; 18 (3): 246-252.

[16] Hizli Sayar GH, Ozten E, Tufan AE, et al. Transcranial magnetic stimulation during pregnancy. Arch Womens Ment Health. DOI 10.1007/s 00737-013-0397-0 (online first publication).

[17] Janicak PG, Nahas Z, Lisanby SH, et al. Durability of clinical benefit with transcranial magnetic stimulation (TMS) in the treatment of pharmacoresistant major depression: assessment of relapse during a 6-month, multisite, open-label study. Brain Stimul. 2010; 3 (4): 187-199.

[18] George MS, Lisanby SH, Avery D, et al. Daily left prefrontal transcranial magnetic stimulation therapy for major depressive disorder: a sham-controlled randomized trial. Arch Gen Psychiatry. 2010; 67 (5): 507-516.

[19] Miller LJ. Use of electroconvulsive therapy during pregnancy. Hospital \& Community Psychiatry. 1994; 45 (5): 444-450. 